\title{
Repulsive and Attractive Interactions between Polystyrene Chains in a Poor Solvent
}

\author{
Mio ORIBE and Takahiro SATO ${ }^{\dagger}$ \\ Department of Macromolecular Science, Osaka University, \\ 1-1 Machikaneyama-cho, Toyonaka 560-0043, Japan
}

(Received April 12, 2004; Accepted May 25, 2004; Published September 15, 2004)

\begin{abstract}
The osmotic compressibility up to high concentrations as well as the second virial coefficient were measured for low molecular weight polystyrenes dissolved in a poor solvent cyclohexane at 35,25 , and $15^{\circ} \mathrm{C}$, by sedimentation equilibrium. The results of the osmotic compressibility over wide concentration ranges were favorably compared with a recently developed thermodynamic perturbation theory based on the spherocylinder model bearing a square-well potential, and from the comparison, the hard-core diameter $d$ and the depth $\varepsilon$ of the attractive square-well potential including in the theory were determined for polystyrene in cyclohexane. Compared with the previous results of $d$ and $\varepsilon$ for the same polymer in $15^{\circ} \mathrm{C}$ toluene (a good solvent), it turned out that $\varepsilon$ increases and $d$ decreases with reducing the solvent quality. [DOI 10.1295/polymj.36.747]

KEY WORDS Polystyrene / Osmotic Compressibility / Second Virial Coefficient / Sedimentation Equilibrium / Thermodynamic Perturbation Theory /
\end{abstract}

The distribution function theories for polymer solutions $^{1-3}$ express the polymer intermolecular interaction in terms of the potential $U_{12}(1,2)$ of mean force, where the arguments 1 and 2 represent all coordinates specifying the position, orientation, and conformation of polymer chains 1 and 2, respectively. If the polymer chain is divided into $N_{0}$ identical (spherical) segments, $U_{12}(1,2)$ may be given by

$$
U_{12}(1,2)=\sum_{i_{1}=1}^{N_{0}} \sum_{i_{2}=1}^{N_{0}} u\left(R_{i_{1} i_{2}}\right)
$$

where $u\left(R_{i_{1} i_{2}}\right)$ is the pair potential of mean force between segments $i_{1}$ and $i_{2}$ belonging to polymer chains 1 and 2, respectively, which is a function of the distance $R_{i_{1} i_{2}}$ of the two segments. For neutral polymers, the potential $u\left(R_{i_{1} i_{2}}\right)$ consists of short-ranged repulsive and long-ranged attractive interaction parts.

The two-parameter theory ${ }^{1-3}$ and also the renormalization-group theory ${ }^{4-7}$ assume that the intermolecular excluded volume effect on the virial coefficients and the osmotic pressure can be expressed as a function of the binary cluster integral $\beta_{2}$ defined by

$$
\beta_{2} \equiv 4 \pi \int_{0}^{\infty}\left\{1-\exp \left[-u(R) / k_{\mathrm{B}} T\right]\right\} R^{2} \mathrm{~d} R
$$

$\left(k_{\mathrm{B}}\right.$ : the Boltzmann constant; $T$ : the absolute temperature) instead of the full function of $u(R)$. However, this assumption does not necessarily hold. Near the theta condition where $\beta_{2}$ vanishes, the ternary cluster integral or the three-segment interaction becomes im- portant in virial coefficients against the two-parameter theory. ${ }^{8}$ When the polymer concentration is beyond the semidilute regime, the osmotic pressure or the osmotic compressibility cannot be described by the renormalization-group theory. ${ }^{9}$

Alternatively, the thermodynamic perturbation theory chooses a system of particles interacting by the hard-core potential as a reference system, and treats the long-ranged attractive interaction in a perturbative way. For example, Barker and Henderson proposed a perturbation theory for the system of spherical particles with a square-well potential. Their theory includes all perturbation terms using the Padé approximation. Recently, Koyama and Sato ${ }^{10}$ extended the Barker-Henderson theory to the wormlike spherocylinder system. In the theory, the intermolecular interaction is characterized in terms of the hardcore diameter $d$ and the depth $\varepsilon$ of the attractive square-well potential, instead of $\beta_{2}$, and the threebody interaction is included in the theory. This theory is however based on the single contact approximation, so that it is applicable only to polymers with a sufficiently small number of Kuhn's statistical segments. Koyama and Sato demonstrated previously that the osmotic compressibility of low molecular weight polystyrenes (lower than ca. 10,000) dissolved in a good solvent toluene can be accurately described by this perturbation theory over a wide range of the polymer concentration.

In the present study, we have examined the applicability of the perturbation theory to low molecular

${ }^{\dagger}$ To whom correspondence should be addressed (E-mail: tsato@chem.sci.osaka-u.ac.jp.). 
weight polystyrenes dissolved in a poor solvent cyclohexane. For the poor solvent system, the attractive interaction among polymers is enhanced and the perturbation term in the theory becomes important. Therefore, we can more critically test the perturbation theory by comparing it with the poor solvent system.

So far, the osmotic compressibility of cyclohexane solutions of polystyrene was investigated over wide ranges of the concentration by several workers ${ }^{11-15}$ However, most of them used rather high molecular weight polystyrene samples, and may not be suitable for the purpose of the present study. On the other hand, the second virial coefficient $A_{2}$ of oligo- and polystyrenes in cyclohexane over a wide molecular weight range was measured by Yamakawa et al. ${ }^{16,17}$ We have utilized their $A_{2}$ data to determine the interaction parameters $d$ and $\varepsilon$ of polystyrene in cyclohexane, as explained below.

\section{EXPERIMENTAL}

Standard polystyrene samples F1, A5000, and A2500 purchased from Tosoh Corp., were divided into several fractions by fractional precipitation using toluene as the solvent and methanol as the precipitant and middle fractions (F1-2, A5000-3, and A2500-5) were used for the following experiments.

Sedimentation equilibrium measurements were made for dilute through concentrated cyclohexane solutions of the polystyrene fractions at 35,25 , and $15^{\circ} \mathrm{C}$, using a Beckman-Coulter Optima XL-I ultracentrifuge, equipped with a Rayleigh interferometer with a 675-nm light emitting from a diode laser. Aluminum 12-mm double-sector cells were used, and the height of the solution column was adjusted to $c a$. $2.5 \mathrm{~mm}$. Rotor speeds were chosen in the range from 5000 to $20000 \mathrm{rpm}$, depending on the polymer concentration. The apparent molecular weight $M_{\text {app }}$ was calculated from the equation

$$
M_{\mathrm{app}}=\frac{2 R T\left(c_{\mathrm{b}}-c_{\mathrm{a}}\right)}{\omega^{2}\left(r_{\mathrm{b}}^{2}-r_{\mathrm{a}}^{2}\right) c_{0}(\partial \rho / \partial c)}
$$

where $r_{\mathrm{b}}$ and $r_{\mathrm{a}}$ are the distance from the center of revolution to the cell bottom and meniscus respectively, $c_{\mathrm{b}}$ and $c_{\mathrm{a}}$ are polymer mass concentrations at $r_{\mathrm{b}}$ and $r_{\mathrm{a}}$ respectively under the centrifugal field which are estimated by interferometry; $\omega$ is the angular velocity, $\rho$ and $c$ are the density and mass concentration of the solution respectively, $c_{0}$ is $c$ at $\omega=0$, and $R$ is the gas constant.

If the concentration difference $\Delta c \equiv c_{\mathrm{b}}-c_{\mathrm{a}}$ is much smaller than the average concentration $\bar{c} \equiv$ $\left(c_{\mathrm{b}}+c_{\mathrm{a}}\right) / 2, M_{\text {app }}$ is related to the reciprocal of the osmotic compressibility $\partial \Pi / \partial c$ at $c=\bar{c}$ by ${ }^{18,19}$

$$
\frac{1}{M_{\text {app }}}=\frac{1}{R T} \frac{\partial \Pi}{\partial c}
$$

On the other hand, for dilute solutions, $M_{\text {app }}$ is written in the form ${ }^{18,19}$

$$
\frac{1}{M_{\mathrm{app}}}=\frac{1}{M_{\mathrm{w}}}+2 A_{2} \bar{c}+O\left(\bar{c}^{2}\right)
$$

where $M_{\mathrm{w}}$ is the weight-average molecular weight, and $A_{2}$ is the second virial coefficient.

Densities $\rho$ and excess refractive indices $\Delta n$ of cyclohexane solutions of the three polystyrene fractions were measured at 35,25 , and $15^{\circ} \mathrm{C}$ as functions of the polymer concentration $c$ to obtain $\partial \rho / \partial c$ and $\partial n / \partial c$ necessary to calculate $M_{\text {app }}$. Detailed procedures are described in the previous paper, ${ }^{10}$ and the results of $\partial \rho / \partial c$ and $\partial n / \partial c$ are listed in Table I.

\section{RESULTS}

Figure 1 shows double logarithmic plots of $(\partial \Pi / \partial c) / R T\left(=M_{\text {app }^{-1}}\right) v s . \bar{c}$ for cyclohexane solutions of three low molecular weight polystyrene fractions at $35^{\circ} \mathrm{C}$ (circles), $25^{\circ} \mathrm{C}$ (triangles), and $15^{\circ} \mathrm{C}$ (diamonds) over wide ranges of the concentration. The concentration dependence of $(\partial \Pi / \partial c) / R T$ is very weak at low $\bar{c}$, but becomes steep at high $\bar{c}$ for all the fractions at all the temperatures. For the fraction A2500-5 with $M_{\mathrm{w}}=2400,(\partial \Pi / \partial c) / R T$ increases with $\bar{c}$ rather smoothly even at the low temperature.

Table I. Characteristics of polystyrene fractions used

\begin{tabular}{lccccc}
\hline Sample & Temperature & $\partial \rho / \partial c$ & $\begin{array}{c}\partial n / \partial c \\
\left(\mathrm{~cm}^{3} \mathrm{~g}^{-1}\right)\end{array}$ & $M_{\mathrm{w}}$ & $\begin{array}{r}A_{2} \\
\left(10^{-4} \mathrm{~cm}^{3} \mathrm{~mol}^{-1} \mathrm{~g}^{-2}\right)\end{array}$ \\
\hline F1-2 & $35^{\circ} \mathrm{C}$ & 0.286 & 0.165 & 10,500 & 0.45 \\
& $25^{\circ} \mathrm{C}$ & 0.279 & 0.162 & 10,500 & -0.75 \\
\multirow{2}{*}{ A5000-3 } & $15^{\circ} \mathrm{C}$ & 0.273 & 0.159 & 10,500 & -2.3 \\
& $35^{\circ} \mathrm{C}$ & 0.283 & 0.159 & 6,000 & 0.6 \\
& $25^{\circ} \mathrm{C}$ & 0.276 & 0.156 & 6,000 & -0.6 \\
$\mathrm{~A} 2500-5$ & $15^{\circ} \mathrm{C}$ & 0.270 & 0.153 & 6,000 & -2.3 \\
& $35^{\circ} \mathrm{C}$ & 0.273 & 0.153 & 2,400 & 0.0 \\
& $25^{\circ} \mathrm{C}$ & 0.267 & 0.150 & 2,400 & -1.8 \\
\hline
\end{tabular}




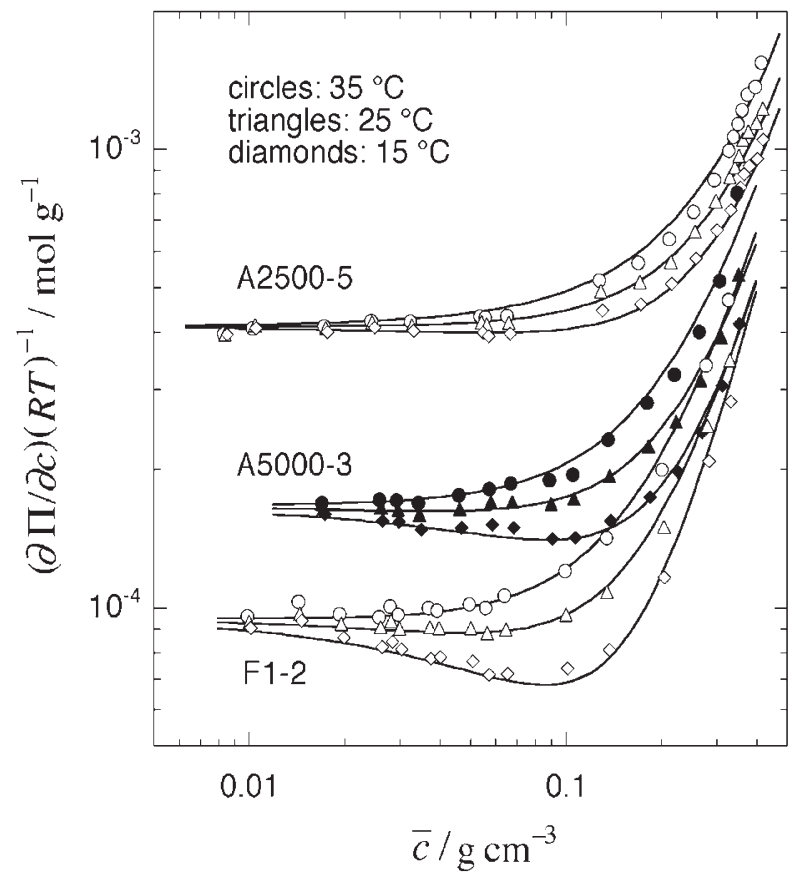

Figure 1. Double logarithmic plot of $(\partial \Pi / \partial c) / R T v s . \bar{c}$ for cyclohexane solutions of three low molecular weight polystyrene fractions at $35^{\circ} \mathrm{C}$ (circles), $25^{\circ} \mathrm{C}$ (triangles), and $15^{\circ} \mathrm{C}$ (diamonds). The solid curves connect the data point smoothly.

On the other hand, $(\partial \Pi / \partial c) / R T$ for the fraction F1-2 with $M_{\mathrm{w}}=10500$ takes a minimum around $\bar{c} \approx 0.1$ $\mathrm{g} / \mathrm{cm}^{3}$ at $15^{\circ} \mathrm{C}$. Similar concentration dependencies of $(\partial \Pi / \partial c) / R T$ for cyclohexane solutions of polystyrene with $M_{\mathrm{w}} \approx 10^{4}$ near the $\theta$ temperature have already been reported previously. ${ }^{14,15}$

From data points at low $\bar{c}$, we have determined $M_{\mathrm{w}}$ and $A_{2}$ for each fraction, using eq 5. The results are listed in Table I. As shown in Figure 2a, our results (filled circles) of $A_{2}$ obtained at 35,25 , and $15^{\circ} \mathrm{C}$ agree with Yamakawa et al.'s results ${ }^{16,17}$ (unfilled circles) in cyclohexane at $34.5,25$, and $15^{\circ} \mathrm{C}$, respectively. In a high $M_{\mathrm{w}}$ region, $A_{2}$ tends to zero at $34.5^{\circ} \mathrm{C}$ (the $\theta$ temperature), and approaches to negative asymptotic values at 25 and $15^{\circ} \mathrm{C}$. Strong molecular weight dependencies of $A_{2}$ in a low $M_{\mathrm{w}}$ region come from the chain end effect as analyzed below.

\section{DISCUSSION}

\section{Theories}

Under the single-contact approximation, we can define a pair of the closest approaching contour points $s_{1}$ and $s_{2}$ on two interacting polymer chains, and the intermolecular interaction potential $u(r)$ can be written as a function of the distance $r$ between $s_{1}$ and $s_{2}$. For the (helical-wormlike) spherocylinder model with a square-well potential, we may use the following $u(r)$ :
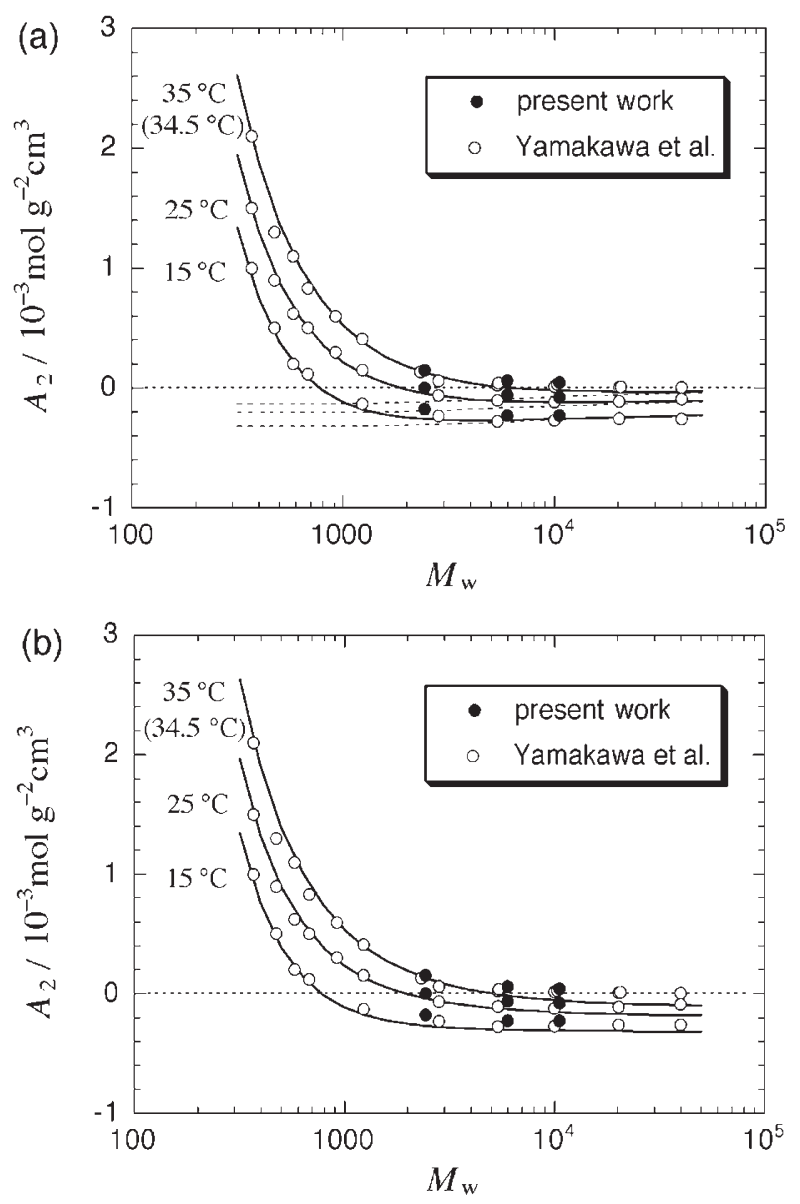

Figure 2. Molecular weight dependence of $A_{2}$ of oligo- and polystyrenes in cyclohexane at different temperature. (a) Filled circles, data obtained in the present study (at 35,25 , and $15^{\circ} \mathrm{C}$ ); unfilled circles, data of Yamakawa et al. ${ }^{16,17}$ (at 34.5, 25, and $15^{\circ} \mathrm{C}$ ); solid curves, $A_{2}$ calculated by eq 16 ; dashed curves, $A_{2}$ calculated by eq 16 without the end-effect terms. (b) symbols, the same as those in Panel a; solid curves, $A_{2}$ calculated by eq 14 with $d+\delta=-0.03 \mathrm{~nm}\left(35,34.5^{\circ} \mathrm{C}\right),-0.05 \mathrm{~nm}\left(25^{\circ} \mathrm{C}\right)$, and $-0.085 \mathrm{~nm}\left(15^{\circ} \mathrm{C}\right)$.

$$
u(r)= \begin{cases}\infty & (0 \leq r<d) \\ -\varepsilon_{\mathrm{X}} & \left(d \leq r<\frac{3}{2} d\right) \\ 0 & \left(\frac{3}{2} d \leq r\right)\end{cases}
$$

where $d$ is the cylinder diameter and $\varepsilon_{\mathrm{X}}$ is the depth of the square well potential. The closest approaching contour points $s_{1}$ and $s_{2}$ are assumed to be on the cylinder axes with a length $L_{\mathrm{c}}: 0 \leq s_{i} \leq L_{\mathrm{c}}(i=1,2)$. If polymer-chain ends are chemically different from the middle chain, $\varepsilon_{\mathrm{X}}$ depends on the relative configuration of the two interacting spherocylinders: $\varepsilon_{\mathrm{X}}=\varepsilon_{\mathrm{ee}}$ when $s_{1}$ and $s_{2}$ equal to 0 or $L_{\mathrm{c}}, \varepsilon_{\mathrm{X}}=\varepsilon_{\mathrm{me}}$ when $s_{1}\left(\right.$ or $\left.s_{2}\right)=$ 0 or $L_{\mathrm{c}}$ and $0<s_{2}\left(\right.$ or $\left.s_{1}\right)<L_{\mathrm{c}}$, and $\varepsilon_{\mathrm{X}}=\varepsilon_{\mathrm{mm}}$ when $s_{1}$ and $s_{2}$ are between 0 and $L_{\mathrm{c}}$.

The thermodynamic perturbation theory ${ }^{10}$ gives the 
reciprocal of the osmotic compressibility $\partial \Pi / \partial c$ of the spherocylinder solution in the form

$$
\frac{\partial \Pi}{\partial c}=\frac{\partial \Pi_{0}}{\partial c}+\frac{\partial \Pi_{\mathrm{w}, \mathrm{mm}}}{\partial c}+\frac{\partial \Pi_{\mathrm{w}, \mathrm{me}}}{\partial c}+\frac{\partial \Pi_{\mathrm{w}, \mathrm{ee}}}{\partial c}
$$

where $\partial \Pi_{0} / \partial c$ is the term for the reference system of the hard spherocylinder with $\varepsilon_{\mathrm{X}}=0$, and $\partial \Pi_{\mathrm{w}, \mathrm{mm}} / \partial c$, $\partial \Pi_{\mathrm{w}, \mathrm{me}} / \partial c$, and $\partial \Pi_{\mathrm{w}, \mathrm{ee}} / \partial c$ are perturbation terms arising from the attractive interactions between chain middle portions, between middle and end portions, and between two chain end portions, respectively. The first term may be formulated by the scaled particle theory for the (isotropic) hard spherocylinder system, of which result is written as ${ }^{20}$

$$
\begin{aligned}
\frac{\partial \Pi_{0}}{\partial c}= & \frac{R T}{M\left(1-v c^{\prime}\right)^{2}} \\
& \times\left[1+B \frac{c^{\prime}}{1-v c^{\prime}}+2 C\left(\frac{c^{\prime}}{1-v c^{\prime}}\right)^{2}\right]
\end{aligned}
$$

where $M, v$, and $c^{\prime}$ are the molar mass, volume, and number concentration of spherocylinders in the solution, respectively. The coefficients $B$ and $C$ are defined by

$$
\begin{aligned}
& B=\frac{\pi}{2} L_{\mathrm{c}}{ }^{2} d+6 v, \\
& C=\left(v+\frac{\pi}{12} d^{3}\right)\left(B-2 v-\frac{\pi}{6} d^{3}\right)
\end{aligned}
$$

The perturbation terms are given by

$$
\begin{aligned}
\frac{\partial \Pi_{\mathrm{w}, \mathrm{X}}}{\partial c}= & \frac{2 R T M_{\mathrm{L}}}{3 d^{2} M^{2}} b_{\mathrm{X}}\left[2 \Psi\left(\hat{\varepsilon}_{\mathrm{X}}\right) c^{\prime *}\right. \\
& \left.+4 \frac{d \Psi\left(\hat{\varepsilon}_{\mathrm{X}}\right)}{d c^{\prime *}} c^{\prime * 2}+\frac{d^{2} \Psi\left(\hat{\varepsilon}_{\mathrm{X}}\right)}{d c^{\prime * 2}} c^{\prime * 3}\right]
\end{aligned}
$$

where $M_{\mathrm{L}}$ is the molar mass per unit contour length of the spherocylinder, and $\hat{\varepsilon}_{\mathrm{X}}, b_{\mathrm{X}}$, and $c^{\prime *}$ are defined by ${ }^{20}$

$$
\begin{aligned}
& \hat{\varepsilon}_{\mathrm{X}} \equiv \frac{\varepsilon_{\mathrm{X}}}{k_{\mathrm{B}} T}, \quad b_{\mathrm{mm}} \equiv \frac{3 L_{\mathrm{c}}^{2} d}{8}, \\
& b_{\mathrm{me}} \equiv \frac{3 L_{\mathrm{c}} d^{2}}{2}, \quad b_{\mathrm{ee}} \equiv d^{3}, \quad c^{*} \equiv \frac{6}{\pi} v c^{\prime}
\end{aligned}
$$

and $\Psi\left(\hat{\varepsilon}_{\mathrm{X}}\right)$ is the function of Barker and Henderson ${ }^{21}$ given by

$$
\Psi\left(\hat{\varepsilon}_{\mathrm{X}}\right) \equiv \frac{P^{(1)} f^{(1)^{2}} \hat{\varepsilon}_{\mathrm{X}}}{f^{(1)}-\frac{1}{2} f^{(2)} \hat{\varepsilon}_{\mathrm{X}}}
$$

with

$$
f^{(i)}=1+Q^{(i)} c^{\prime *}+\frac{C^{(i)}}{c^{* *}}\left[1-\exp \left(-\frac{\alpha^{(i)} c^{\prime *}}{\beta^{(i)}-c^{* *}}\right)\right]
$$

$$
-\frac{\alpha^{(i)} C^{(i)}}{\beta^{(i)}} \quad(i=1,2)
$$

The parameters $Q^{(i)}, C^{(i)}, \alpha^{(i)}$, and $\beta^{(i)}$ in eq 13 , as well as $P^{(1)}$ in eq 12 are given in Table I of ref 10 . The functions $f^{(1)}$ and $f^{(2)}$ are related to the first and second order perturbations, respectively. ${ }^{21}$

From the virial expansion of eq 7, the second virial coefficient $A_{2}$ is written as

$$
\begin{aligned}
A_{2}= & \frac{\pi N_{\mathrm{A}}}{4 M_{\mathrm{L}}^{2}}\left[d+\delta+\left(\frac{8}{3} d+\delta^{\prime}\right) \frac{\mathrm{d} M_{\mathrm{L}}}{M}\right. \\
& \left.+\left(\frac{4}{9} d+\delta^{\prime \prime}\right)\left(\frac{\mathrm{d} M_{\mathrm{L}}}{M}\right)^{2}\right]
\end{aligned}
$$

where $N_{\mathrm{A}}$ is the Avogadro constant, and $\delta, \delta^{\prime}$, and $\delta^{\prime \prime}$ are defined by ${ }^{20}$

$$
\begin{aligned}
& \frac{\delta}{d}=\frac{3 P^{(1)} \hat{\varepsilon}_{\mathrm{mm}}}{\pi\left(2-\hat{\varepsilon}_{\mathrm{mm}}\right)}, \quad \frac{\delta^{\prime}}{d}=\frac{12 P^{(1)} \hat{\varepsilon}_{\mathrm{me}}}{\pi\left(2-\hat{\varepsilon}_{\mathrm{me}}\right)}-\frac{4}{3} \frac{\delta}{d}, \\
& \frac{\delta^{\prime \prime}}{d}=\frac{8 P^{(1)} \hat{\varepsilon}_{\mathrm{ee}}}{\pi\left(2-\hat{\varepsilon}_{\mathrm{ee}}\right)}-\frac{4}{9} \frac{\delta}{d}-\frac{2}{3} \frac{\delta^{\prime}}{d}
\end{aligned}
$$

It is noted that eq 14 is based on the single-contact approximation.

On the other hand, $A_{2}$ can be formulated also by the cluster expansion theory for the helical wormlike beads model. ${ }^{3,22,23}$ Retaining the first order terms of the binary and ternary cluster integrals, we can write

$$
\begin{aligned}
A_{2}= & \frac{N_{\mathrm{A}} c_{\infty}^{3 / 2}}{2 M_{\mathrm{L}}^{2}}\left[B_{2}+2\left(\frac{3}{2 \pi}\right)^{3 / 2} \lambda^{2} B_{3} I(N)\right] \\
& +\frac{a_{2,1}}{M}+\frac{a_{2,2}}{M^{2}}
\end{aligned}
$$

where $c_{\infty}$ is the characteristic ratio, $B_{2}$ and $B_{3}$ are the excluded-volume strengths of the two- and three-segment interactions, ${ }^{3}$ proportional to the binary and ternary cluster integrals, respectively, $\lambda^{-1}$ is the stiffness parameter, and $a_{2,1}$ and $a_{2,2}$ are the parameters characterizing interchain interactions between the chain middle and end portions and between two chain end portions, respectively. The function $I(N)$ of the reduced contour length $N\left(=\lambda M / M_{\mathrm{L}}\right)$ was recently calculated by Yamakawa and Yoshizaki. ${ }^{23}$ In eq 16, the $B_{2}$ term is the single-contact one, and the $B_{3}$ term represents the three-segment interaction between two chains which involves two segments of one chain and one segment of the other chain interacting at a point (a special case of double-contact interactions). The double-contact term with $B_{3}$ is not included in eq 14 .

Finally, the cluster expansion theory for the beads model gives the third virial coefficient $A_{3}{ }^{0}$ at the $\theta$ condition as ${ }^{3}$ 


$$
A_{3}{ }^{0}=\frac{N_{\mathrm{A}}{ }^{2} c_{\infty}^{3}}{3 M_{\mathrm{L}}{ }^{3}} B_{3}
$$

This equation, however, does not include the polymer chain end effect, so that it may be applicable only at sufficiently high molecular weights.

\section{Comparison between Theory and Experiment}

As explained above, the intermolecular interaction between polymer chains is characterized in terms of the hard-core diameter $(d)$ and the depths $\left(\varepsilon_{\mathrm{mm}}, \varepsilon_{\mathrm{me}}\right.$, and $\varepsilon_{\mathrm{ee}}$ ) of the square-well potential in the perturbation theory, and of the excluded-volume strengths $\left(B_{2}\right.$ and $\left.B_{3}\right)$ and the end-effect parameters $\left(a_{2,1}\right.$ and $\left.a_{2,2}\right)$ in the cluster expansion theory. To determine those parameters, we also need the helical wormlike chain parameters, the molar mass per unit contour length $M_{\mathrm{L}}$, the characteristic ratio $c_{\infty}$, and the stiffness parameter $\lambda^{-1}$, all of which have been already determined for polystyrene in cyclohexane to be $358 \mathrm{~nm}^{-1}$, 0.816 , and $2.06 \mathrm{~nm}$, respectively. ${ }^{3}$

Nakamura et al. ${ }^{8}$ and Yamakawa et al. ${ }^{24}$ measured the third virial coefficient $A_{3}{ }^{0}$ of polystyrene in cyclohexane at $34.5^{\circ} \mathrm{C}$ (the $\theta$ temperature), from which an asymptotic value of $A_{3}{ }^{0}$ was estimated to be $4.7 \times$ $10^{-4} \mathrm{~cm}^{6} \mathrm{~mol} / \mathrm{g}^{3}$. This result gives a value of 0.33 $\mathrm{nm}^{3}$ for $B_{3}$ using eq 17 , which is smaller than that in toluene $\left(=1.4 \mathrm{~nm}^{3}\right) .{ }^{10}$ The two-parameter theory cannot explain this residual three-segment interaction at the $\theta$ condition.

As shown in Figure 2a, $A_{2}$ obtained by Yamakawa et $a l .{ }^{16,17}$ and in the present study are independent of the molecular weight at $M_{\mathrm{w}} \gtrsim 5,000$ for the three temperatures, and the asymptotic values can be identified with $\left(N_{\mathrm{A}} c_{\infty}^{3 / 2} / 2 M_{\mathrm{L}}{ }^{2}\right)\left[B_{2}+2(3 / 2 \pi)^{3 / 2} \lambda^{2} B_{3} I(\infty)\right]$ in eq 16 , where $I(\infty)=1.465 .{ }^{23}$ Furthermore, Nakamura et $a{ }^{25}$ demonstrated that $B_{3}$ of polystyrene is almost independent of temperature in a poor solvent, so that we can estimate $B_{2}$ at the three temperatures from the asymptotic values of $A_{2}$ and the above $B_{3}$ value at the $\theta$ temperature. The results are listed in Table II.

Table II. Interaction parameters of polystyrene in cyclohexane $(\mathrm{CH})$ and toluene (TOL)

\begin{tabular}{lcccc}
\hline & $\begin{array}{c}\mathrm{CH} \\
\left(34.5,35^{\circ} \mathrm{C}\right)\end{array}$ & $\begin{array}{c}\mathrm{CH} \\
\left(25^{\circ} \mathrm{C}\right)\end{array}$ & $\begin{array}{c}\mathrm{CH} \\
\left(15^{\circ} \mathrm{C}\right)\end{array}$ & $\begin{array}{c}\mathrm{TOL}^{\mathrm{a}} \\
\left(15^{\circ} \mathrm{C}\right)\end{array}$ \\
\hline$B_{2} / \mathrm{nm}$ & -0.075 & -0.12 & -0.185 & 0.74 \\
$B_{3} / \mathrm{nm}^{3}$ & 0.33 & $0.33^{\mathrm{b}}$ & $0.33^{\mathrm{b}}$ & 1.4 \\
$a_{2,1} / \mathrm{cm}^{3} \mathrm{~g}^{-1}$ & 0.55 & 0.30 & 0.05 & 2.0 \\
$a_{2,2} / \mathrm{cm}^{6} \mathrm{~mol}^{-1}$ & 100 & 120 & 150 & -150 \\
$d / \mathrm{nm}$ & 0.45 & 0.425 & 0.43 & 0.56 \\
$\varepsilon_{\mathrm{mm}} / 10^{-21} \mathrm{~J}$ & 1.6 & 1.6 & 1.6 & 0.72 \\
$\varepsilon_{\mathrm{me}} / 10^{-21} \mathrm{~J}$ & 0.82 & 1.1 & 1.4 & -0.68 \\
$\varepsilon_{\mathrm{ee}} / 10^{-21} \mathrm{~J}$ & -0.72 & -1.0 & -0.97 & 0.60 \\
\hline
\end{tabular}

${ }^{\mathrm{a}}$ Results obtained previously. ${ }^{10,20 \mathrm{~b}}$ Assumed.
Because the $\theta$ condition is defined as the state where the two-segment and three-segment interactions cancel out in the present cluster expansion theory, ${ }^{23,26,27} B_{2}$ itself does not vanish at the $\theta$ temperature.

The remaining end-effect parameters $a_{2,1}$ and $a_{2,2}$ were determined so as to lead best fits of eq 16 to the $A_{2}$ data in the lower molecular weight region, which are also listed in Table II. The values of $a_{2,1}$ and $a_{2,2}$ are slightly larger and smaller, respectively, than the results ${ }^{17}$ obtained without considering the effect of the three-segment interaction. The solid curves in Figure 2a represent the values calculated by eq 16 with the parameters thus determined. On the other hand, the dashed curves in the same figure represent the theoretical $A_{2}$ without the end-effect terms in eq 16. The end effect becomes important at $M_{\mathrm{w}} \lesssim$ 5,000 .

Substituting the end-effect terms determined above into eq 14 , we searched values of $d+\delta$ leading to best fits of eq 14 to the $A_{2}$ data in a lower $M_{\mathrm{w}}$ region. Figure $2 \mathrm{~b}$ shows the best fit results by the solid curves, which slightly deviate downward from the data points at $M_{\mathrm{w}} \gtrsim 10^{4}$. The deviations arise from the effect of the double contact including in eq 16 (the $B_{3}$ term) but not in eq 14. Corresponding to $B_{2}$ in eq 16 , the value of $d+\delta$ and thus the asymptotic value of the theoretical $A_{2}$ at the $\theta$ temperature is slightly negative, as seen from the top solid curve in Figure $2 \mathrm{~b}$. The disagreement between the thermodynamic perturbation theory using the single-contact approximation and experiment with respect to $A_{2}$ was observed in the good solvent toluene too, ${ }^{10}$ but it is less remarkable in the poor solvent cyclohexane. This may be due to smaller contributions of multiple-contact effects expressed in terms of power series of $B_{2}$ and $B_{3}$ to $A_{2}$, because of smaller $\left|B_{2}\right|$ and $B_{3}$ in the poor solvent ( $c f$. Table II).

To separate the value of $d$ from $d+\delta$ thus determined, we compared theoretical values of $(\partial \Pi / \partial c) /$ $R T$ calculated by eqs 7,8 , and 10 for different values of $d$ with experimental results for low molecular weight polystyrene up to high polymer concentrations shown in Figure 1. If a value of $d$ is chosen, $\delta, \delta^{\prime}$, and $\delta^{\prime \prime}$ can be determined from $d+\delta, a_{2,1}$, and $a_{2,2}$ ( $c f$. eqs 14 and 16), and $\varepsilon_{\mathrm{mm}}, \varepsilon_{\mathrm{me}}$, and $\varepsilon_{\mathrm{ee}}$ can be calculated from $\delta, \delta^{\prime}$, and $\delta^{\prime \prime}$ using eq 15 . As shown by the solid curves in Figure 3 , the values of $d$ and also $\varepsilon_{\mathrm{mm}}$, $\varepsilon_{\text {me }}$, and $\varepsilon_{\text {ee }}$ listed in Table II closely fit eqs 7,8 , and 10 to the experimental $(\partial \Pi / \partial c) / R T$ for different molecular weights over the wide concentration range. Therefore, the perturbation theory using the singlecontact approximation is valid for expressing the osmotic compressibility for flexible polymers with low molecular weights dissolved in the poor solvent. The previous study ${ }^{10}$ demonstrated that the same theory was favorably compared with the osmotic compressi- 

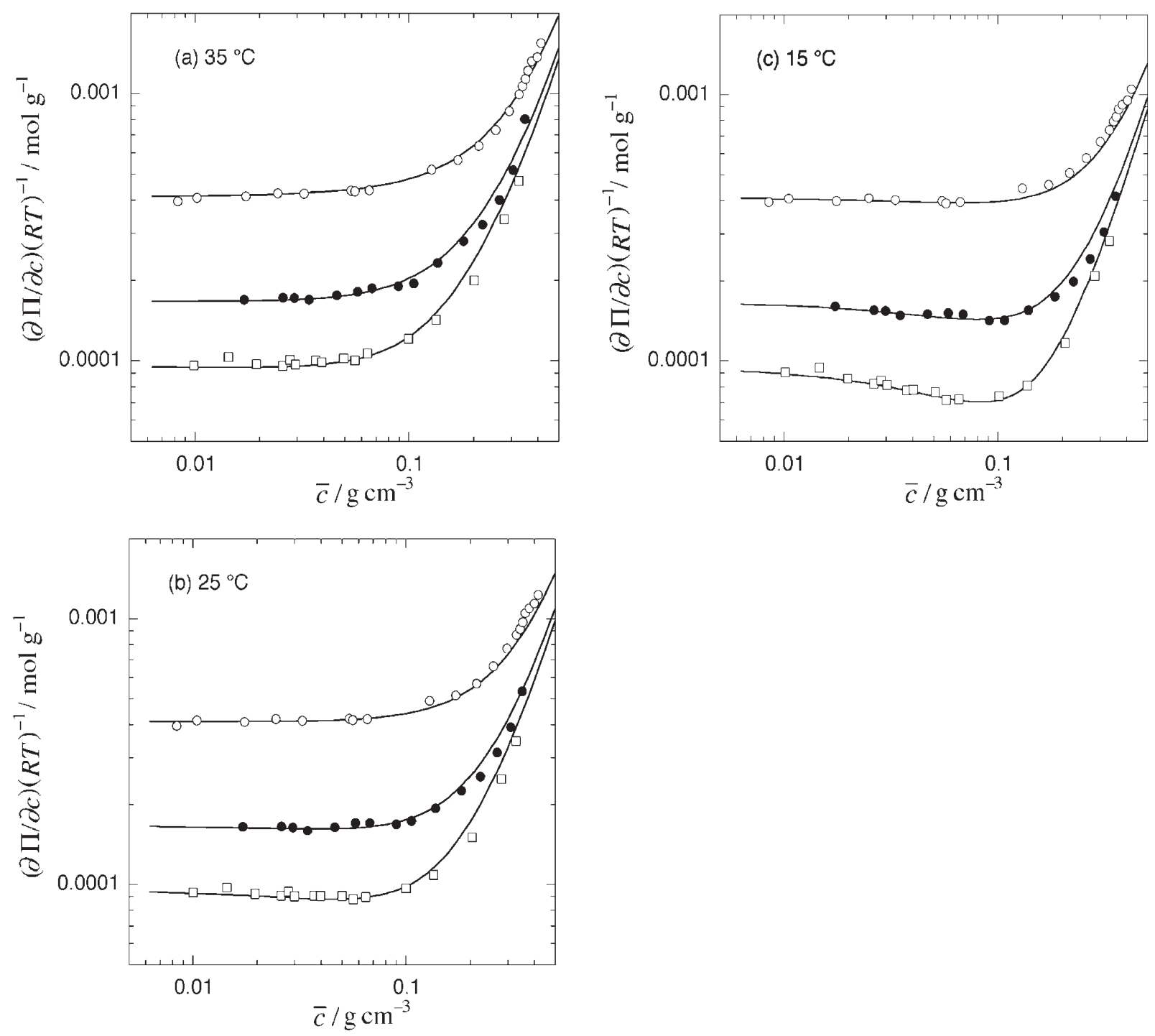

Figure 3. Comparison of $(\partial \Pi / \partial c) / R T$ for cyclohexane solutions of low molecular weight polystyrene fractions at $35^{\circ} \mathrm{C}$ (unfilled circles), $25^{\circ} \mathrm{C}$ (filled circles), and $15^{\circ} \mathrm{C}$ (squares) with theoretical ones calculated by eqs 7,8 , and 10 with the interaction parameters listed in Table II (solid curves).

bility for toluene solutions of polystyrene with $M_{\mathrm{w}} \lesssim 10^{4}$. Therefore, we can say that the perturbation theory has a wide applicability with respect to the solvent quality.

Table II compares the interaction parameters of polystyrene in toluene determined previously ${ }^{10}$ and in cyclohexane. The values of $\varepsilon_{\mathrm{mm}}$ in cyclohexane are independent of temperature, but larger than that in toluene, i.e., the attractive force between polystyrene chains is stronger in cyclohexane as expected. Since the thermal energy diminishes with decreasing temperature, the attractive potential in cyclohexane plays more important roles in solution properties at lower temperatures. The signs of $\varepsilon_{\mathrm{me}}$ and $\varepsilon_{\mathrm{ee}}$ indicate that the interactions between the middle and end portions and between end portions of polystyrene chains are attractive and repulsive, respectively, in cyclohex- ane, both of which are opposite in the case of the toluene solution. It may be possible for $\varepsilon_{\mathrm{ee}}$ to be negative, if the initiation and termination ends of the polystyrene chain have different chemical structures.

Although the hard-core diameter $d$ in cyclohexane is almost constant at different temperatures, it is considerably smaller than that in toluene. Since the interaction parameter $d$ characterizes the potential of mean force, the solvent intermediating between interacting two polymer chains may implicitly affect $d$. At present, we know little the relationship between the potential of mean force and bare potentials of the polymerpolymer, polymer-solvent, and solvent-solvent pairs. Our result indicates that the repulsive interaction range of the potential of mean force between polymer chains diminishes with decreasing the solvent quality, but its theoretical justification is left in future work. 
Acknowledgment. We are grateful to Dr. Yo Nakamura and Prof. Takashi Norisuye at Osaka University, Toyonaka, Japan for valuable comments about the second and third virial coefficients, and also to Prof. Takenao Yoshizaki at Kyoto University, Kyoto, Japan for informing on ref 23.

\section{REFERENCES}

1. H. Yamakawa, "Modern Theory of Polymer Solutions," Harper \& Row, New York, N.Y., 1971.

2. H. Fujita, "Polymer Solutions," Vol 9, Elsevier, Amsterdam, 1990.

3. H. Yamakawa, "Helical Wormlike Chains in Polymer Solutions," Springer-Verlag, Berlin and Heidelberg, 1997.

4. P.-G. de Gennes, "Scaling Concepts in Polymer Physics," Cornell Univ. Press, Ithaca, N.Y., 1979.

5. Y. Oono, Adv. Chem. Phys., 61, 301 (1985).

6. K. F. Freed, "Renormalization Group Theory of Macromolecules," John Wiley \& Sons, New York, N.Y., 1987.

7. J. des Cloizeaux and G. Jannink, "Polymers in Solution. Their Modelling and Structure," Clarendon Press, Oxford, U.K., 1990.

8. Y. Nakamura, T. Norisuye, and A. Teramoto, Macromolecules, 24, 4904 (1991).

9. I. Noda, Y. Higo, N. Ueno, and T. Fujimoto, Macromolecules, 17, 1055 (1984).

10. R. Koyama and T. Sato, Macromolecules, 35, 2235 (2002).

11. T. G. Scholte, Eur. Polym. J., 6, 1063 (1970).

12. T. G. Scholte, J. Polym. Sci., Part A-2: Polym. Phys., 8, 841 (1970).

13. T. G. Scholte, J. Polym. Sci., Part A-2: Polym. Phys., 9,
1553 (1971).

14. Z. Tong, S. Ohashi, Y. Einaga, and H. Fujita, Polym. J., 15, 835 (1983).

15. Y. Einaga, S. Ohashi, Z. Tong, and H. Fujita, Macromolecules, 17, 527 (1984).

16. Y. Einaga, F. Abe, and H. Yamakawa, Macromolecules, 26, 6243 (1993).

17. H. Yamakawa, F. Abe, and Y. Einaga, Macromolecules, 27, 5704 (1994).

18. H. Fujita, "Foundation of Ultracentrifugal Analysis," Wiley-Interscience, New York, N.Y., 1975.

19. M. Kurata, "Thermodynamics of Polymer Solutions," Harwood Academic Publishers, Chur, 1982.

20. There are errors in the previous paper. In eq $2.4, B=$ $(\pi / 2) L_{\mathrm{c}}^{2} d \rho$; eqs 2.6 and 2.8 , the excess free energies should be divided by $k_{\mathrm{B}} T$; in eq $2.9, b_{\mathrm{me}}=3 L_{\mathrm{c}} d^{2} / 2$; in eq 2.11 , the term with $C$ should be multiplied by 2 ; in eq $2.14, \delta^{3}$ should be replaced by $\delta_{3}$; in eqs 2.16 and $2.17,3 \varepsilon_{\mathrm{me}}$ should be replaced by $6 \varepsilon_{\text {me }}$; in eq $2.19,108$ should be replaced by 216 ; in Table 3, $\quad \varepsilon_{\mathrm{me}} / k_{\mathrm{B}} T=2 \pi\left(4 \delta+3 \delta^{\prime}\right) /\left[36 P^{(1)} d+\pi(4 \delta+\right.$ $\left.\left.3 \delta^{\prime}\right)\right]=-0.17, \delta_{3}^{\prime}=1.4 \mathrm{~nm}$, and $\delta_{3}^{\prime \prime}=-1.9 \mathrm{~nm}$.

21. J. A. Barker and D. Henderson, Rev. Mod. Phys., 48, 587 (1976).

22. H. Yamakawa, Macromolecules, 25, 1912 (1992).

23. H. Yamakawa and T. Yoshizaki, J. Chem. Phys., 119, 1257 (2003).

24. H. Yamakawa, F. Abe, and Y. Einaga, Macromolecules, 27, 3272 (1994).

25. Y. Nakamura, N. Inoue, T. Norisuye, and A. Teramoto, Macromolecules, 30, 631 (1997).

26. H. Yamakawa, J. Chem. Phys., 45, 2606 (1966).

27. T. Norisuye and Y. Nakamura, Macromolecules, 27, 2054 (1994). 